\title{
Validating Protocol Composition for Progress by Parallel Step Reachability Analysis
}

\author{
Gurdip Singh* \\ Dept. of Computing and Information Sciences, Kansas State University \\ 234 Nichols Hall, Manhattan, KS 66506, singh@cis.ksu.edu
}

Hong Liu

Bellcore Applied Research, MCC 1J-222R

445 South Street, Morristown, NJ 07960, lhong@bellcore.com

\begin{abstract}
In this paper, we propose a parallel step exploration technique for protocol validation in the context of protocol composition. A protocol is modeled as a network of extended communicating finite state machines (ECFSM's). A composite protocol is defined as an interleaved execution of a set of component protocols subject to a set of constraints such as synchronization, ordering and inhibition. By encoding the constraints into the component protocols and the analysis algorithm, our method keeps each process in the component protocols as a separate entity and performs validation without constructing the composite protocol explicitly. We show that our technique not only achieves significant state reduction but also preserves the progress property of the composite protocol in the reduced state space. To our best knowledge, this is the first attempt to adapt existing state reduction techniques to the validation of protocol composition.
\end{abstract}

\section{INTRODUCTION}

Designing a correct protocol is a challenging task due to the complex interactions among communicating entities. One way to tackle the complexity in protocol design and analysis is through composition, where one divides the functionality of a protocol into subfunctions, develops component protocols for the subfunctions, and then combines them to obtain the composite protocol for the original problem. [CGL85, CM86, LT93, S94a] discuss methods for constructing a multiphase protocol,whereas [Lin88, Lin91, S93, S94b] study techniques for constructing protocols which performed multiple functions at the same time.

All these techniques impose sufficient conditions on the component protocols so that properties of the composite protocol can be inferred from those of the component protocols (which are smaller in size and therefore easier to analyze). While the analysis of the composite protocol is avoided, the sufficient conditions restrict the class of protocols that can be composed - one might still be able to construct correct protocols from a set of component protocols which do not satisfy those conditions. In this setting, the composite protocol needs to be validated for correctness.

Many techniques have been proposed to tackle the state explosion problem in protocol validation by eliminating redundant interleaving of independent transitions in different processes during state exploration. (Informally, two transitions are independent if they cannot enable or disable each

\footnotetext{
* This work was supported by NSF under grants CCR9211621 and CCR9502506.
} 
other; otherwise they are dependent.) The partial order based techniques [V90, HGW92, GW93, GW94, P93, P94, LM96c] select a representative sequential execution for each set of equivalent transition sequences, while other techniques allow more than one process to make progress in a single step [YG82, RW82, II83, GH85, ZB86, CR93, OU94, LM96a, LM96b, LM96c, SU96].

In this paper, a protocol is modeled as a set of extended communicating finite state machines (ECFSM's). A composite protocol is then modeled as an interleaved execution of a set of component protocols subject to a set of constraints such as synchronization, ordering and inhibition. The ordering constraint was used for sequential composition in [S94a], while the synchronization constraint was used for parallel composition in [S93, S94b]. These constraints can be combined to produce a variety of composite protocols, such as serial-parallel compositions. Other than those constraints, we impose no additional restrictions on the component protocols.

Although existing techniques can potentially reduce the state space drastically, they might not be most effective if applied to the composite protocol directly. Suppose we construct $R$ from two component protocols $P$ and $Q$, and a set of constraints by composing $P_{i}$ and $Q_{i}$ into $R_{i}$ at each site $i$. By definition, independent transitions can only come from different processes. However, in constructing $R_{i}$ from $P_{i}$ and $Q_{i}$, we are in fact putting many originally independent transitions between $P_{i}$ and $Q_{i}$ into $R_{i}$ to make them artificially dependent for subsequent validation.

To achieve greater state reduction, we propose a modification to the parallel step reachability analysis [OU94] that keeps $P_{i}$ and $Q_{i}$ as separate entities so that both can make progress in parallel during state exploration. This provides us with a much larger set of independent actions and allows us to exploit concurrency between actions in the component protocols. By encoding the constraints into each process and the validation algorithm, we are able to enforce the composition constraints on-the-fly. We show that our technique significantly reduces the state space explored while preserves the progress property of the composite protocol.

The rest of the paper is organized as follows: Section 2 introduces the extended communicating finite state machines as the model for protocol specification; Section 3 formally specifies the composition constraints and presents a algorithm to construct a composite protocol. Our parallel step reachability analysis technique is described in Section 4. An example is given Section 5. Conclusion and future work are given in Section 6. Due to space limitations, we only outline the algorithms and omit the proofs of theorems. Please refer to the full paper [SL97] for details.

\section{THE ECFSM MODEL}

A communication protocol $P$ is modeled as a network of $n \geq 2$ extended communicating finite state machines (ECFSM's), denoted as $P=P_{1}\left\|P_{2}\right\| \ldots \| P_{n}$. Each $P_{i}$ is a finite state machine with local variables, denoted as $\left(A_{i}, V_{i}, X_{i}, T_{i}, x_{i}^{0}\right)$, where $A_{i}$ is a finite set of actions, $V_{i}$ is a finite set of local variables, $X_{i}$ is a finite set of local states, $T_{i}$ is the transition relation, and $x_{i}^{0}$ is the initial local state. Processes exchange messages via uni-directional FIFO channels. The channel from $P_{i}$ to $P_{j}$ is denoted as $C_{i j}$, bounded by a positive integer $B_{i j}$. The content of $C_{i j}$ is denoted as $c_{i j} \cdot c_{i j}=\epsilon$ if $C_{i j}$ is empty. Define first $\left(c_{i j}\right)=m$ if $c_{i j}=m \cdot c_{i j}^{\prime}$; first $\left(c_{i j}\right)=\epsilon$ if $c_{i j}=\epsilon$.

An action $a \in A_{i}$ is of the form en $(a) \longrightarrow a$, where en $(a)$ is a boolean function on $V_{i}$, called the enabling condition of $a$, and $a$ is the associated computation consisting of a non-empty sequence of statements separated by ";". A statement is either a local statement involving only local variables, a send statement $P_{j} ! m$ appending $m$ at the end of channel $C_{i j}$, or a receive statement $P_{j}$ ? $m$ removing $m$ from the head of channel $C_{j i}$ if $f i r s t\left(c_{j i}\right)=m$. We assume that each action contains 
at most one send or receive statement. We omit the enabling condition if it is identically true. The transition relation $T_{i}$ consists of tuples of the form $\left(s_{i}, a, s_{i}^{\prime}\right)$, also denoted as $s_{i} \stackrel{a}{\longrightarrow} s_{i}^{\prime}$ or $\operatorname{succ}\left(s_{i}, a\right)=s_{i}^{\prime} . T_{i}$ is assumed to be deterministic but can be partially defined. We usually refer to transition $\left(s_{i}, a, s_{i}^{\prime}\right)$ as transition $a$ defined at $s_{i}$, or transition $a$ if $s_{i}$ is clear from the context.

Let $\left\langle v_{i}\right\rangle$ be the tuple of values of the local variables of $V_{i}$. A state of $P_{i}$ is defined as $s_{i}=\left(x_{i},<v_{i}>\right)$. The initial state of $P_{i}$ is denoted as $\left.s_{i}^{0}=\left(x_{i}^{0},<v_{i}^{0}\right\rangle\right)$, where $\left.<v_{i}^{0}\right\rangle$ are the initial values of the local variables. We assume that each local variable has a finite domain. So each $P_{i}$ has a finite number of states. A global state of $P$ is defined as $S_{P}=\left(\left\langle s_{i}\right\rangle,\left\langle c_{i j}\right\rangle\right)$, where $s_{i}$ denotes the state of $P_{i}$ and $c_{i j}$ denotes the content of channel $C_{i j}$. The initial global state of $P$ is denoted as $S_{P}^{0}=\left(\left\langle s_{i}^{0}\right\rangle,\left\langle\epsilon_{i j}\right\rangle\right)$, where $\epsilon_{i j}$ denotes $c_{i j}=\epsilon$.

Given a global state $\left.\left.S_{P}=\left(<s_{i}\right\rangle_{,}<c_{i j}\right\rangle\right)$ and a transition $a$ defined at $s_{i}$, let stmt be a statement of $a$. stmt is enabled in $S_{P}$ iff (1) en(a) is true in $S_{P} ;(2)$ if $s t m t=P_{j} ! m$ then $\left|c_{i j}\right|<B_{i j}$ in $S_{P}$; and (3) if stmt $=P_{j} ? m$ then $c_{j i}=m \cdot c_{j i}^{\prime}$ in $S_{P}$. Transition $a$ is enabled in $S_{P}$ if all the statements in $a$ are enabled in $S_{P}$; otherwise it is disabled in $S_{P}$. The set of enabled and disabled transitions in $S_{P}$ are denoted as enabled $\left(P_{i}, S_{P}\right)$ and $\operatorname{disabled}\left(P_{i}, S_{P}\right)$, respectively.

The execution of $a$ in $S_{P}$ is assumed to be atomic. If $a$ is executed, it will result in a global state $S_{P}^{\prime}$ of $P$ such that (1) $s_{i}^{\prime}=\operatorname{succ}\left(s_{i}, a\right) ;(2) c_{i j}^{\prime}=c_{i j} \cdot m$ if a contains a send statement $P_{j} ! m ;(3) c_{j i}=m \cdot c_{j i}^{\prime}$ if $a$ contains a receive statement $P_{j}$ ? $m$; and (4) the rest of the elements in $S_{P}^{\prime}$ remain the same as those in $S_{P}$. We say $S_{P}^{\prime}$ is directly reachable from $S_{P}$ via a, denoted as $S_{P} \stackrel{a}{\longrightarrow} S_{P}^{\prime}$ or $S_{P}^{\prime}=\operatorname{succ}\left(S_{P}, a\right) . S_{P}^{\prime}$ is directly reachable from $S_{P}$, denoted as $S_{P} \mapsto S_{P}^{\prime}$, iff $S_{P}^{\prime}=\operatorname{succ}\left(S_{P}, a\right)$ for some action $a$. Denote $\mapsto^{*}$ as the reflexive, transitive closure of $\mapsto$. $S_{P}^{\prime}$ is reachable from $S_{P}$ iff $S_{P} \mapsto^{*} S_{P}^{\prime}$. When $S_{P}=S_{P}^{0}, S_{P}^{\prime}$ is a reachable global state. A reachable global state $S_{P}$ is non-progress if there is no transition enabled in $S_{P}$. The set of reachable global states of $P$ is denoted as $\mathbf{R}_{P}$.

Suppose $S_{P} \mapsto^{*} S_{P}^{\prime}$, an execution sequence from $S_{P}$ to $S_{P}^{\prime}$ is a finite sequence $e x \triangleq Z_{P}^{0} \stackrel{t_{1}}{\longrightarrow}$ $Z_{P}^{1} \stackrel{t_{2}}{\longrightarrow} \ldots \stackrel{t_{k}}{\longrightarrow} Z_{P}^{k}, k \geq 0$, such that $Z_{P}^{0}=S_{P}, Z_{P}^{k}=S_{P}^{\prime}$, and $\forall h, 1 \leq h \leq k: Z_{P}^{h}=$ $\operatorname{succ}\left(Z_{P}^{h-1}, t_{h}\right)$. When $S_{P}=S_{P}^{0}$, ex is called an execution sequence for $S_{P}^{\prime}$. The length of ex is defined as the number of transitions in $e x$, denoted as $|e x|=k \geq 0$. The set of execution sequences from $S_{P}^{0}$ is denoted as behaviors $(P)$.

Two transitions $a$ and $b$ are independent in a global state $S_{P}$ if: (1) If $a$ is enabled in $S_{P}$, then $b$ is enabled in $S_{P}$ iff it is also enabled in $\operatorname{succ}\left(S_{P}, a\right)$; and (2) If $b$ is enabled in $S_{P}$, then $a$ is enabled in $S_{P}$ iff it is also enabled in $\operatorname{succ}\left(S_{P}, b\right)$; and (3) If both $a$ and $b$ are enabled in $S_{P}$, then $\operatorname{succ}\left(\operatorname{succ}\left(S_{P}, a\right), b\right)=\operatorname{succ}\left(\operatorname{succ}\left(S_{P}, b\right), a\right)$. Otherwise, $a$ and $b$ are dependent. By definition, all transitions in the same process are dependent.

Since we assume that each local variable in $P_{i}$ has a finite domain, each channel has a finite capacity, and a send statement is blocked if the destination channel is full, it follows that $\mathbf{R}_{P}$ is finite. As a result, it is decidable whether $P$ has the required progress property.

\section{COMPOSITION OF PROTOCOLS}

The composite protocol $R$ from $P$ and $Q$ is defined as an interleaved execution of $P$ and $Q$ at each site subject to a set of constraints. Without loss of generality, we make the following assumptions: (1) $P$ and $Q$ have the same number of processes, with $P_{i}$ and $Q_{i}$ running at site $i$. $R_{i}$ is constructed from $P_{i}$ and $Q_{i}$ and a set of constraints on their actions. (2) The send and receive statements 
from $P_{i}$ and $Q_{i}$ operate on the same set of channels in $R_{i}$. So each send statement $P_{j} ! m\left(Q_{j} ! m^{\prime}\right)$ from $P_{i}\left(Q_{i}\right)$ is renamed as $R_{j} ! m\left(R_{j} ! m^{\prime}\right)$, and each receive statement $P_{j} ? m\left(Q_{j} ? m^{\prime}\right)$ from $P_{i}$ $\left(Q_{i}\right)$ is renamed as $R_{j} ? m\left(R_{j} ? m^{\prime}\right) ;(3)$ The message sets of $P_{i}$ and $Q_{i}$ are disjoint, and so are the local variable sets. This can be ensured through proper renaming; (4) The bound on a channel in $R$ is the sum of the bounds on the same channel in $P$ and $Q$.

\subsection{Specifying the Constraints}

We first define a cross product operator $\times$ for $P_{i}$ and $Q_{i}$. Let $P_{i}=\left(p A_{i}, p V_{i}, p X_{i}, p T_{i}, p x_{i}^{0}\right)^{*}$ and $Q_{i}=\left(q A_{i}, q V_{i}, q X_{i}, q T_{i}, q x_{i}^{0}\right)$ Then $G_{i}=P_{i} \times Q_{i}$ is an ECFSM $\left(g A_{i}, g V_{i}, g X_{i}, g T_{i}, g x_{i}^{0}\right)$ such that $g A_{i}=p A_{i} \cup q A_{i}, g V_{i}=p V_{i} \cup q V_{i}, g X_{i}=\left\{\left(p x_{i}, q x_{i}\right) \mid\left(p x_{i} \in p X_{i}\right) \wedge\left(q x_{i} \in q X_{i}\right)\right\}$ and $g x_{i}^{0}=\left(p x_{i}^{0}, q x_{i}^{0}\right)$. A state of $G_{i}$ is denoted as $g s_{i}=\left(g x_{i},<v_{i}>\right)$. The initial state of $G_{i}$ is denoted as $g s_{i}^{0}=\left(g x_{i}^{0},\left\langle v_{i}^{0}\right\rangle\right) . g s_{i}$ and $g s_{i}^{0}$ can be rewritten as $\left(p s_{i}, q s_{i}\right)$ and $\left(p s_{i}^{0}, q s_{i}^{0}\right)$, respectively. $g T_{i}$ consists of tuples of the form $\left(g s_{i}, c, g s_{i}^{\prime}\right)$, where $g s_{i}=\left(p s_{i}, q s_{i}\right)$ and $g s_{i}^{\prime}=\left(p s_{i}^{\prime}, q s_{i}^{\prime}\right)$, such that if $c \in p A_{i}$, then $\left(p s_{i}, c, p s_{i}^{\prime}\right) \in p T_{i}$ and $q s_{i}^{\prime}=q s_{i}$; if $c \in q A_{i}$, then $\left(q s_{i}, c, q s_{i}^{\prime}\right) \in q T_{i}$ and $p s_{i}^{\prime}=p s_{i}$.

Let $G=G_{1}\left\|G_{2}\right\| \ldots \| G_{n}$ be the resulting protocol, denoted as $G=P \times Q$. The set of constraints on $P_{i}$ and $Q_{i}$ are imposed on the set of behaviors of $G$. We have identified three types of constraints: synchronization, ordering and inhibition. These constraints are specified as pairs of actions $(a, b)$ or $(b, a)$, where $a$ and $b$ are actions of $P_{i}$ and $Q_{i}$, respectively. Let $e x \triangleq S_{G}^{0} \stackrel{t_{1}}{\longrightarrow} S_{G}^{1} \stackrel{t_{2}}{\longrightarrow} \ldots \stackrel{t_{k}}{\longrightarrow} S_{G}^{k}$ be an execution sequence of $G$, where $S_{G}^{0}$ is the initial global state of $G$. Let $a^{x}$ and $b^{x}$ be the $x^{\text {th }}$ occurrence of $a$ and $b$ in ex, respectively; and $l_{a}$ and $l_{b}$ be the number of occurrences of $a$ and $b$ in $e x$, respectively.

- ex satisfies the synchronization constraint $(a, b)$ between $P_{i}$ and $Q_{i}$ if $(1)\left|l_{a}-l_{b}\right| \leq 1$; and (2) $\forall x, 1 \leq x \leq \min \left(l_{a}, l_{b}\right)$ : Let $t_{h}=a^{x}$ and $t_{l}=b^{x}$, if $h<l$ then both $a$ and $b$ are enabled in $S_{G}^{h-1}$ and $\forall j, h<j<l: t_{j}$ is not an action of $P_{i}$ or $Q_{i}$. A similar condition must hold if $l<h$; and (3) If $l_{a}>l_{b}$ and the last occurrence of $a$ in $e x$ is $t_{h}$, then both $a$ and $b$ are enabled in $S_{G}^{h-1}$ and $\forall j, h<j \leq k: t_{j}$ is not an action of $P_{i}$ or $Q_{i}$. A similar condition must hold for $l_{b}>l_{a}$. The set of synchronization constraints of $P_{i}$ and $Q_{i}$ is denoted as $\operatorname{synch}\left(P_{i}, Q_{i}\right)$.

- ex satisfies the ordering constraint $(a, b)$ from $P_{i}$ to $Q_{i}$ if $0 \leq l_{a}-l_{b} \leq 1$ and $\forall x, 1 \leq x \leq l_{b}$ : (1) If $b^{x}=t_{h}$ then $\exists t_{l}, l<h: t_{l}=a^{x}$; and (2) if $a^{x}=t_{h}$ and $x>1$ then $\exists t_{l}, l<h: t_{l}=b^{x}-1$. The set of ordering constraints from $P_{i}$ to $Q_{i}$ is denoted as $\operatorname{order}\left(P_{i}, Q_{i}\right)$. The set of ordering constraints from $Q_{i}$ to $P_{i}$, denoted as $\operatorname{order}\left(P_{i}, Q_{i}\right)$, can be defined similarly.

- ex satisfies the inhibition constraint $(a, b)$ from $P_{i}$ to $Q_{i}$ if the following condition is satisfied: If $t_{h}=a^{1}$ then there is no $t_{l}$ such that $l>h$ and $t_{l}=b^{x}$ for any $x \geq 1$. The set of inhibition constraints from $P_{i}$ to $Q_{i}$ is denoted as inhibit $\left(P_{i}, Q_{i}\right)$. The set of inhibition constraints from $Q_{i}$ to $P_{i}$, denoted as inhibit $\left(Q_{i}, P_{i}\right)$, can be defined similarly.

Let constraints $\left(P_{i}, Q_{i}\right)$ be the set of constraints imposed on site $i$. Then constraint $(P, Q)=$ $\bigcup_{i=1}^{n}$ constraints $\left(P_{i}, Q_{i}\right)$ is the set of constraints for composing $P$ and $Q$. Even though they are defined with respect to (w.r.t) a finite execution sequence, they also apply to infinite behaviors of $G$. We say an infinite execution sequence satisfies a constraint if every prefix of the sequence satisfies the constraint. Note that while the set of synchronization constraints is a symmetric relation, the sets of ordering and inhibition constraints are not. For the latter two types, we need to distinguish the cases where $P_{i}$ takes precedence over $Q_{i}$ from those where $Q_{i}$ takes precedence

\footnotetext{
* We add a prefix $p$ to all the elements of protocol $P$. The same convention applies to protocols $G, H, Q$ and $R$.
} 
over $P_{i}$. We impose the following four requirements for constraints $\left(P_{i}, Q_{i}\right)$ to be well-specified for site $i$ : (1) The set of synchronization, ordering and inhibition constraints be mutually disjoint. (2) Each transition of $P_{i}$ be synchronized with at most one transition of $Q_{i}$ and vice versa. This restriction avoids cases during execution where a transition of $P_{i}\left(Q_{i}\right)$ has to be synchronized with more than one transition of $Q_{i}\left(P_{i}\right)$ in the same global state of the composite protocol. (3) If $a$ and $b$ are synchronized, they should not both contain receive statements expecting messages from the same channel, since at any global state of $G$, only one of the two receive statements will be enabled. (4) There be no cyclic dependency in the sets of ordering constraints and inhibition constraints $t$. constraints $(P, Q)$ is well-specified iff each constraints $\left(P_{i}, Q_{i}\right)$ is well-specified. In the rest of the paper, unless otherwise specified, we assume that constraints $(P, Q)$ is well-specified.

\subsection{Constructing the Composite Protocol}

The construction of $R_{i}$ from $P_{i}, Q_{i}$ and constraints $\left(P_{i}, Q_{i}\right)$ is composed of three steps. The first step introduces a set of new variables for each constraint. The next step adds new conjuncts and/or local statements to the transitions in $P_{i}$ and $Q_{i}$. The last step computes $R_{i}$ from $P_{i} \times Q_{i}$ by deleting and modifying those transitions involved in the synchronization constraints.

Step 1: we introduce a new local variable for each constraint $(a, b)$ or $(b, a)$ on $P_{i}$ and $Q_{i}$ as follows: (1) $\forall(a, b) \in \operatorname{synch}\left(P_{i}, Q_{i}\right)$, add $\operatorname{syn}_{i}^{a b} ;(2) \forall(a, b) \in \operatorname{order}\left(P_{i}, Q_{i}\right)$, add $\operatorname{ord}_{i}^{a b} ;(3) \forall(b, a) \in$ $\operatorname{order}\left(Q_{i}, P_{i}\right)$, add ord od $_{i} ;(4) \forall(a, b) \in \operatorname{inhibit}\left(P_{i}, Q_{i}\right)$, add inh $h_{i}^{a b} ;(5) \forall(b, a) \in \operatorname{inhibit}\left(Q_{i}, P_{i}\right)$, add $i n h_{i}^{b a}$. Except for $s y n_{i}^{a b}$, which is a three value $\{0,1,2\}$ variable with initial value 0 , all other variables are boolean variables with initial value false.

Let $\operatorname{syn} V_{i}$, ord $V_{i}$ and $i n h V_{i}$ be the sets of synchronization, ordering and inhibition variables introduced, respectively. Let $g s y n_{i}$ be the conjuction of the propositions $s y n_{i}^{a b}=0$ for all synchronization variables $s y n_{i}^{a b}$ created above. For each $(a, b) \in \operatorname{synch}(a, b)$, let $g s y n_{i}^{a b}$ be the proposition that is formed by deleting conjunct $s y n_{i}^{a b}=0$ from gsyn $_{i}$.

Step 2: We modify each action in $P_{i}$ and $Q_{i}$ by adding conjunct(s) and/or local statement(s) to its enabling condition and computation. Specifically, for each $a \in p A_{i}$ and $b \in q A_{i}$, we modify $a$ and $b$ as follows:

(1) $a(b)$ is not involved in any constraint in constraints $\left(P_{i}, Q_{i}\right)$. Then add $g s y n_{i}$ to en(a) $(e n(b))$ as a conjunct if it is not already there.

(2) $(a, b) \in \operatorname{synch}\left(P_{i}, Q_{i}\right)$. Then for $a$, add $g s y n_{i}^{a b}$ and $e n(b)$ as conjunts to en $(a)$ and add statement ${ }^{\mathrm{if}} s y n_{i}^{a b}=0$ then $\operatorname{syn}_{i}^{a b}:=1$ else $\operatorname{syn}_{i}^{a b}:=0^{n}$ to its computation. For $b$, add $g s y n_{i}^{a b}$ and $e n(a)$ as conjuncts to $e n(b)$ and add statement ${ }^{\mu}$ if $s y n_{i}^{a b}=0$ then $s y n_{i}^{a b}:=2$ else syn $_{i}^{a b}:=0^{n}$ to its computation.

(3) $(a, b) \in \operatorname{order}\left(P_{i}, Q_{i}\right)$. Then for $a$, add conjunct $\neg$ ord $d_{i}^{a b}$ to en $(a)$ and add statement "ord $d_{i}^{a b}:=$ true to its computation. For $b$, add conjunct $\operatorname{ord}_{i}^{a b}$ to en(b) and add statement "ord $d_{i}^{a b}:=$ false $e^{n}$ to its computation. The case for $(b, a) \in \operatorname{order}\left(Q_{i}, P_{i}\right)$ can be carried out similarly.

(4) $(a, b) \in$ inhibit $\left(P_{i}, Q_{i}\right)$. Then for $a$, add statement ${ } i n h_{i}^{a b}:=t r u e^{n}$ to its computation. For $b$, add conjunct $\neg i n h_{i}^{a b}$ to $e n(b)$. The case for $(b, a) \in$ inhibit $\left(Q_{i}, P_{i}\right)$ can be carried out similarly.

Intuitively, item (3) enforces the ordering constraints; and item (4) implements the inhibition constraints. However, items (1) and (2) together only partly enforce the synchronization constraints. The missing part will be filled in the next step.

$\dagger_{\text {Please refer to the full paper for how these cyclic dependency can be statically checked. }}$ 
Step 9: We compute $R_{i}$ from $P_{i} \times Q_{i}$ as follows: for each $(a, b) \in \operatorname{synch}\left(P_{i}, Q_{i}\right)$, for each transition $t_{a}=\left(p x_{i}, a, p x_{i}^{\prime}\right)$ in $P_{i}$ and $t_{b}=\left(q x_{i}, b, q x_{i}^{\prime}\right)$ in $Q_{i}$, let $r x_{i}^{1}=\left(p x_{i}, q x_{i}\right), r x_{i}^{2}=\left(p x_{i}^{\prime}, q x_{i}\right), r x_{i}^{3}=$ $\left(p x_{i}, q x_{i}^{\prime}\right)$, and $r x_{i}^{4}=\left(p x_{i}^{\prime}, q x_{i}^{\prime}\right)$, then except for these four states, $t_{a}$ and $t_{b}$ are removed from any other states in $R_{i}$ as outgoing transitions. There are four cases to consider:

(1) $\left(p x_{i}=p x_{i}^{\prime}\right) \wedge\left(q x_{i}=q x_{i}^{\prime}\right)$. Both $t_{a}$ and $t_{b}$ are self loops in $P_{i}$ and $Q_{i}$, respectively. The two corresponding transitions in $R_{i}$ are also self loops: $\left(r x_{i}^{1}, a, r x_{i}^{1}\right)$ and $\left(r x_{i}^{1}, b, r x_{i}^{1}\right)$. We add a conjunct $s y n_{i}^{a b} \neq 1$ to en $(a)$ and a conjunct $s y n_{i}^{a b} \neq 2$ to $e n(b)$.

(2) $\left(p x_{i} \neq p x_{i}^{\prime}\right) \wedge\left(q x_{i}=q x_{i}^{\prime}\right) . t_{a}$ is not a self loop in $P_{i}$ but $t_{b}$ is a self loop in $Q_{i}$. The corresponding transition for $t_{a}$ in $R_{i}$ is $\left(r x_{i}^{1}, a, r x_{i}^{2}\right)$ and $e n(a)$ is enhanced with a new conjunct $s y n_{i}^{a b} \neq 1$. The corresponding transitions of $t_{b}$ in $R_{i}$ are $\left(r x_{i}^{1}, b, r x_{i}^{1}\right)$ and $\left(r x_{i}^{2}, b, r x_{i}^{2}\right)$, both of which are self loops. We add a conjunct $s y n_{i}^{a b}=0$ to $e n(b)$ of the first transition and a conjunct $s y n_{i}^{a b}=1$ to en $(b)$ of the second one.

(3) $\left(p x_{i}=p x_{i}^{\prime}\right) \wedge\left(q x_{i} \neq q x_{i}^{\prime}\right) . t_{a}$ is a self loop in $P_{i}$ but $t_{b}$ is not a self loop in $Q_{i}$. The corresponding transitions for $t_{a}$ in $R_{i}$ are $\left(r x_{i}^{1}, a, r x_{i}^{1}\right)\left(r x_{i}^{3}, a, r x_{i}^{3}\right)$, both of which are self loops. We add a conjunct $s y n_{i}^{a b}=0$ to en $(a)$ of the first transition and a conjunct $s y n_{i}^{a b}=2$ to $e n(a)$ of the second one. The corresponding transition of $t_{b}$ in $R_{i}$ is $\left(r x_{i}^{1}, b, r x_{i}^{3}\right)$ and en(b) is enhanced with a new conjunct $s y n_{i}^{a b} \neq 2$.

(4) $\left(p x_{i} \neq p x_{i}^{\prime}\right) \wedge\left(q x_{i} \neq q x_{i}^{\prime}\right)$. Neither $t_{a}$ nor $t_{b}$ is a self loop in $P_{i}$ or $Q_{i}$, respectively. The corresponding transitions of $t_{a}$ in $R_{i}$ are $\left(r x_{i}^{1}, a, r x_{i}^{2}\right)$ and $\left(r x_{i}^{3}, a, r x_{i}^{4}\right)$. We add a new conjunct $s y n_{i}^{a b}=0$ to $e n(a)$ in the first transition and a new conjunct $s y n_{i}^{a b}=2$ to en(a) in the second one. The corresponding transitions of $t_{b}$ in $R_{i}$ are $\left(r x_{i}^{1}, b, r x_{i}^{3}\right)$ and $\left(r x_{i}^{2}, b, r x_{i}^{4}\right)$. We add a new conjunct $s y n_{i}^{a b}=0$ to $e n(b)$ in the first transition and a new conjunct $s y n_{i}^{a b}=1$ to $e n(b)$ in the second one.

\section{End of Algorithm}

Let $\left.S_{R}=\left(<r s_{i}\right\rangle,\left\langle c_{i j}\right\rangle\right)$ be a global state of $R$. Then $r s_{i}=\left(\left(p s_{i}, q s_{i}\right),\left\langle s v_{i}\right\rangle_{i}<o v_{i}\right\rangle$ ,$\left.\left\langle i v_{i}\right\rangle\right)$, where $\left\langle s v_{i}\right\rangle,\left\langle o v_{i}\right\rangle$, and $\left\langle i v_{i}\right\rangle$ are local variables values of $s y n V_{i}$, ord $V_{i}$, and inh $V_{i}$, respectively. The initial global state of $R$ is denoted as $S_{R}^{0}=\left(<r s_{i}^{0}>,<\epsilon_{i j}>\right)$. Here $r s_{i}^{0}=\left(\left(p s_{i}^{0}, q s_{i}^{0}\right),\left\langle s v_{i}^{0}\right\rangle,\left\langle o v_{i}^{0}\right\rangle,\left\langle i v_{i}^{0}\right\rangle\right)$, where $\left.\left\langle s v_{i}^{0}\right\rangle,<o v_{i}^{0}\right\rangle$, and $<i v_{i}^{0}>$ are initial values of variables of syn $V_{i}$ (all 0$)$, ord $V_{i}$ (all false) and inh $V_{i}$ (all false), respectively. Let $e x$ be an execution sequence of $R$ and $\left.e x\right|_{R_{i}}$ be the projection of $e x$ on $r A_{i}$. Clearly, ex satisfies constraints $\left(P_{i}, Q_{i}\right)$ iff ex $\left.\right|_{R_{i}}$ satisfies each constraint specified in constraints $\left(P_{i}, Q_{i}\right)$. It can be shown that each $R_{i}$ thus constructed does ensure that $R$ satisfies constraints $(P, Q)$.

Theorem $1 \forall$ ex $\in$ behaviors $(R): \forall i:\left.e x\right|_{R_{i}}$ satisfies constraints $\left(P_{i}, Q_{i}\right)$.

\section{PARALLEL STEP REACHABILITY ANALYSIS}

There are two major sources that cause dependency between a transition $a$ in $P_{i}$ and a transition $b$ in $Q_{i}:(1)$ If $(a, b)$ or $(b, a)$ belongs to constraints $\left(P_{i}, Q_{i}\right), a$ and $b$ cannot be executed in arbitrary order except for synchronization; (2) If both $a$ and $b$ involve sending a message to the same channel, the channel content will differ by the order in which $a$ and $b$ are executed. To take into account these dependency, we encode constraints $\left(P_{i}, Q_{i}\right)$ into $P_{i}$ and $Q_{i}$, as was done Step 1 and 2 in Section 3 , except that for each $(a, b) \in \operatorname{synch}\left(P_{i}, Q_{i}\right)$, we add a conjunct $\operatorname{syn} n_{i}^{a b} \neq 1$ to $e n(a)$ and $s y n_{i}^{a b} \neq 2$ to en(b). Then instead of constructing $R_{i}$ explicitly in Step 3, we view $P_{i}$ 
and $Q_{i}$ as two threads of process $H_{i}$ in a hypothetical protocol $H=H_{1}\left\|H_{2}\right\| \cdots \| H_{n}$ that share the same set of channels and local variables. We then apply parallel step state exploration to validate $H$, where both $P_{i}$ and $Q_{i}$ can make progress from a global state. Finally, we show that the hypothetical protocol $H$ and the composite protocol $R$ have the same progress property.

\subsection{The Hypothetical Protocol $H$}

A global state of $H$ is denoted as $\left.S_{H}=\left(\left\langle h s_{i}\right\rangle_{,}<c_{i j}\right\rangle\right)$, where $h s_{i}$ is of the form $\left(p s_{i}, q s_{i}\right)$. Since $h s_{i}$ has the same component structure as $r s_{i}$, so are $S_{H}$ and $S_{R}$. Define $S_{H}=\alpha\left(S_{R}\right)$ (or $\left.S_{R}=\alpha^{-1}\left(S_{H}\right)\right)$ iff $S_{H}$ and $S_{R}$ have the same component values. $\alpha$ is a one-to-one mapping from the set of global states in $R$ and the set of global states in $H$. Since a transition is enabled in $\alpha\left(S_{R}\right)$ if it is enabled in $S_{R}, \alpha$ is a homomorphism from $\mathbf{R}_{R}$ to $\mathbf{R}_{H}$ w.r.t the reachability relation $\mapsto^{*}$. Let $\alpha\left(\mathbf{R}_{R}\right)$ be the image of $\mathbf{R}_{R}$ in $\mathbf{R}_{H}$. Then $\alpha\left(\mathbf{R}_{R}\right) \subseteq \mathbf{R}_{H}$.

Suppose $S_{H}=\alpha\left(S_{R}\right)$. It can be shown that $S_{H}$ is reachable via an execution sequence ex in $H$ if $S_{R}$ is reachable via ex in $R$, by induction on $|e x|$. However, the converse is not always true. The main reason is that the additional conjuncts we put on actions $a$ and $b$ for $(a, b) \in \operatorname{synch}\left(P_{i}, Q_{i}\right)$ are not sufficient to ensure that $a$ (or $b$ ) occurs first iff $b$ (or $a$ ) occurs next. For example, consider case (4) in Step 3. At $r x_{i}^{2}$, the conjunct added to $e n(b)$ is $s y n^{a b} \neq 2$ instead of $s y n_{i}^{a b}=1$. When $s y n_{i}^{a b}=0, b$ can be enabled at $r x_{i}^{2}$ in $H$ without executing $a$ at $r x_{i}^{1}$.

To avoid the above situation, we select a subset of enabled $\left(P_{i}, S_{H}\right)$, denoted as enabled $p_{p}\left(P_{i}, S_{H}\right)$. A transition $a \in \operatorname{enabled}_{p}\left(P_{i}, S_{H}\right)$ iff (1) $a$ is not involved in any synchronization constraint, or (2) $(a, b) \in \operatorname{synch}\left(P_{i}, Q_{i}\right)$ and $b \in \operatorname{enabled}\left(Q_{i}, S_{H}\right)$, or $(3)(a, b) \in \operatorname{synch}\left(P_{i}, Q_{i}\right), \operatorname{enabled}\left(P_{i}, S_{H}\right)=$ $\{a\}$, enabled $\left(Q_{i}, S_{H}\right)=\emptyset$, and $s y n_{i}^{a b}=2 . a$ is called a valid transition of $P_{i}$ in $S_{H}$. Similarly, a transition $b \in$ enabled $_{p}\left(Q_{i}, S_{H}\right)$ iff (1) $b$ is not involved in any synchronization constraint, or (2) $(a, b) \in \operatorname{synch}\left(P_{i}, Q_{i}\right)$ and $a \in \operatorname{enabled}\left(P_{i}, S_{H}\right)$, or $(3)(a, b) \in \operatorname{synch}\left(P_{i}, Q_{i}\right), \operatorname{enabled}\left(P_{i}, S_{H}\right)=\emptyset$, enabled $\left(Q_{i}, S_{H}\right)=\{b\}$, and $s y n_{i}^{a b}=1 . b$ is called a valid transition of $Q_{i}$ in $S_{H}$.

Suppose $S_{H}=\alpha\left(S_{R}\right)$. It can be shown that enabled $_{p}\left(P_{i}, S_{H}\right) \cup \operatorname{enabled}_{p}\left(Q_{i}, S_{H}\right)=\operatorname{enabled}\left(R_{i}\right.$, $\left.S_{R}\right)$. Define $\mathbf{R}_{H}^{p}$ as the set of global states reachable from $S_{H}^{0}$ via only valid transitions. If $S_{H} \in \mathbf{R}_{H}^{p}$ and $e x$ is an execution sequence for $S_{H}$ composed of only valid transitions, then $e x$ is also an execution sequence for $S_{R}$. On the other hand, if $e x$ is an execution sequence for $S_{R}$, then it is also an execution sequence for $S_{H}$ with only valid transitions. Hence $\mathbf{R}_{H}^{p}=\alpha\left(\mathbf{R}_{R}\right)$, i.e., $\alpha$ is an isomorphism from $\mathbf{R}_{R}$ to $\mathbf{R}_{H}^{p}$ w.r.t $\mapsto^{*}$. In particular, a non-progress global state $S_{R}$ is reachable in $R$ iff $S_{H}=\alpha\left(S_{R}\right)$ is reachable in $H$ by valid transitions only. So to study the progress property of $R$, it is sufficient to only generate $\mathbf{R}_{H}^{p}$. In the following, we are going to show that it actually suffices to generate only a subset of $\mathbf{R}_{H}^{p}$ via parallel step state exploration.

\subsection{Parallel Step State Exploration}

We partition the set of transitions for $P_{i}$ defined in a global state $S_{H}$ as follows. First, the set of enabled transitions is divided into two sets: local_enabled $\left(P_{i}, S_{H}\right)$ and global_enabled $\left(P_{i}, S_{H}\right)$. Transition $a \in$ enabled $\left(P_{i}, S_{H}\right)$ is locally enabled if $a$ does not contain a send statement that sends a message to the same channel as another transition $b \in \operatorname{enabled}\left(Q_{i}, S_{H}\right)$; otherwise it is globally enabled. Then the set of disabled transitions is also partitioned into two sets: local_disabled $\left(P_{i}, S_{H}\right)$ and global_disabled $\left(P_{i}, S_{H}\right)$. A transition $a \in \operatorname{disabled}\left(P_{i}, S_{H}\right)$ is locally disabled if en $(a)=$ false, or $e n(a)=$ true, $a$ contains a receive statement $P_{j}$ ? $m$, and $\left(\right.$ first $\left.\left(c_{j i}\right)=m^{\prime} \neq m\right) \wedge\left(m^{\prime} \in p M_{j i}\right)$; otherwise it is globally disabled. Let local_enabled $p\left(P_{i}, S_{H}\right)$ and global__enabled ${ }_{p}\left(P_{i}, S_{H}\right)$ be the 
set of locally and globally enabled valid transitions of $P_{i}$ in $S_{H}$, respectively. The transitions for $Q_{i}$ in $S_{H}$ can be partitioned similarly into these four sets.

To define parallel progress, we first compute $U_{i}$, the set of valid transition pairs for $P_{i}$ and $Q_{i}$ in $S_{H}$ from transitions in enabled ${ }_{p}\left(P_{i}, S_{H}\right) \cup$ global disabled $\left(P_{i}, S_{H}\right)$ of $P_{i}$ and enabled $p\left(Q_{i}, S_{H}\right) \cup$ global_disabled $\left(Q_{i}, S_{H}\right)$ of $Q_{i} \ddagger$ There are four cases to consider:

(1) For each $a \in \operatorname{enabled}_{p}\left(P_{i}, S_{H}\right)$ and $b \in \operatorname{enabled}_{p}\left(Q_{i}, S_{H}\right),(a, b) \in U_{i}$, i.e. $P_{i}$ and $Q_{i}$ can execute $a$ and $b$ in parallel if $(i)(a, b) \in \operatorname{synch}\left(P_{i}, Q_{i}\right)$ and $a$ and $b$ do not send messages to the same channel, or (ii) neither $a$ nor $b$ is involved in any synchronization or inhibition constraint. Otherwise, $(a, \lambda),(\lambda, b) \in U_{i}$, where $\lambda$ is a null transition, indicating no progress.

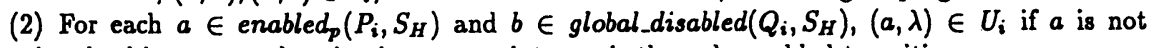
involved in any synchronization constraint or $a$ is the only enabled transition.

(3) For each $a \in$ global_disabled $\left(P_{i}, S_{H}\right)$ and $b \in$ enabled $_{p}\left(Q_{i}, S_{H}\right),(\lambda, b) \in U_{i}$ if $b$ is not involved in any synchronization constraint or $b$ is the only enabled transition.

(4) If global_disabled $\left(P_{i}, S_{H}\right) \neq \emptyset$ and global_disabled $\left(Q_{i}, S_{H}\right) \neq \emptyset$, then $(\lambda, \lambda) \in U_{i}$.

Note that when $(a, b) \in \operatorname{synch}\left(P_{i}, Q_{i}\right)$ but both $a$ and $b$ send messages to the same channel, only one of them can be executed. Suppose $a$ (or $b$ ) is chosen, then in the following global state, $s y n_{i}^{a b}=1$ (or $s y n_{i}^{a b}=2$ ). Hence $g s y n_{i}=$ false, which implies that only $b$ (or $a$ ) can be enabled. In this case, even $b$ (or $a$ ) is not paired with $a$ (or $b$ ), it should still be chosen to execute. Also, when $(a, b) \in$ inhibit $\left(P_{i}, Q_{i}\right)$, even though both are enabled, only one of them can be executed.

Now let $\overrightarrow{\mathbf{u}}$ be a $2 n$-tuple $\left(\left\langle p u_{i}, q u_{i}\right\rangle\right)$ such that $\left(p u_{i}, q u_{i}\right) \in U_{i} . \overrightarrow{\mathbf{u}}$ is a parallel progress vector in $S_{H}$ iff $\exists i:\left(p u_{i}, q u_{i}\right) \neq(\lambda, \lambda)$. Hence in a parallel progress vector, at least one thread must make progress. Since all the non-null transitions in $\vec{u}$ are independent of each other, the resulting global state $S_{H}^{\prime}$ is the same irrespective of the order of execution. In this case, we say that $S_{H}^{\prime}$ is directly parallel reachable from $S_{H}$ via $\overrightarrow{\mathrm{u}}$, denoted as $S_{H} \stackrel{\overrightarrow{\mathrm{u}}}{\longrightarrow} S_{H}^{\prime}$ or $S_{H}^{\prime}=\operatorname{succ}\left(S_{H}, \overrightarrow{\mathrm{u}}\right)$. With this, we can define parallel reachability relations $\mapsto_{p}$ and $\mapsto_{p}^{*}$, and parallel execution sequence accordingly. Denote $\mathbf{P R}_{H}$ as the set of parallel reachable states in $H$ and behaviors $s_{p}(H)$ as the set of parallel execution sequences from $S_{H}^{0}$ in $H$.

Let linear $(\overrightarrow{\mathbf{u}})$ be the set of permutations on the non-null transitions in $\overrightarrow{\mathbf{u}}$. Let $\overrightarrow{\mathbf{u}}_{1}, \overrightarrow{\mathbf{u}}_{2}, \ldots, \overrightarrow{\mathbf{u}}_{k}$, $k \geq 0$, be the sequence of progress vectors in a parallel execution sequence pex. Then linear (pex) is defined as $\{\epsilon\}$ if $k=0$; and as linear $\left(\vec{u}_{1}\right) \cdot \operatorname{linear}\left(\vec{u}_{2}\right) \cdots \operatorname{linear}\left(\overrightarrow{\mathbf{u}}_{k}\right)$ if $k \geq 1$, where $\cdot$ is generalized to handle two sets of sequences, i.e., $A \cdot B=\{a \cdot b \mid(a \in A) \wedge(\bar{b} \in B)\}$. Since each $e x \in \operatorname{linear}(p e x)$ is an execution sequence from $S_{H}$ to $S_{H}^{\prime}$ composed of only valid transitions, $\mathbf{P R}_{H} \subseteq \mathbf{R}_{H}^{p}$. Moreover, pex satisfies constraints $(P, Q)$ iff $\forall$ ex $\in \operatorname{linear}($ pex $):$ ex satisfies constraints $(P, Q)$. Since each $e x$ is also an execution sequence for $S_{R}=\alpha^{-1}\left(S_{H}\right)$ in $R$, by Theorem 1, ex satisfies constraints $(P, Q)$ in $R$.

Theorem $2 \forall$ pex $\in$ behaviors $s_{p}(H):$ pex satisfies constraints $(P, Q)$.

Now suppose $S_{H} \in \mathbf{R}_{H}^{p}, S_{H}$ is a pseudo non-progress global state iff it has no valid transitions. From the above discussion, we know that $S_{H}$ is pseudo non-progress global state in $H$ iff $\alpha^{-1}\left(S_{H}\right)$ is a non-progress global state in $R$. Hence we only need to focus on $\mathbf{S N R}_{H}$, the set of pseudo non-progress global states in $H$. On the other hand, suppose $S_{H} \in \mathbf{P R}_{H}, S_{H}$ is a parallel nonprogress global state in $H$ iff it has no parallel progress vectors. By construction, $S_{H}$ has no parallel progress vectors iff it has no valid transitions. Hence $S_{H}$ is also a pseudo non-progress global state in $H$. Let $\mathbf{P N R}_{H}$ be the set of parallel non-progress global state in $H$. Then $\mathbf{P N R}_{H} \subseteq \mathbf{S N R}_{H}$.

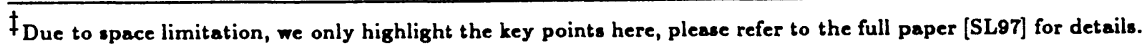


To show the converse, let $e x$ be an execution sequence for a pseudo non-progress global state $S_{H}$. Each transition in $e x$ is a valid transition. Denote $p e_{i}=\left.e x\right|_{P_{i}}$ and $q e_{i}=\left.e x\right|_{Q_{i}}$. Then $\left.\left(<p e_{i}, q e_{i}\right\rangle\right)$ is a local execution sequence set for $S_{H}$. From $\left.\left(<p e_{i}, q e_{i}\right\rangle\right)$, we construct a parallel execution sequence pex for $S_{H}$ as follows. Starting from $S_{H}^{0}$, in Step $k \geq 0$, for each $i$, we compute $\left(p u_{i}^{k+1}, q u_{i}^{k+1}\right)$ for $P_{i}$ and $Q_{i}$ in global state $S_{H}^{k}$ based on the transitions from $p e_{i}$ and $q e_{i}$ in $S_{H}^{k}$. Since ex has only a finite number of transitions, the algorithm must terminate in a finite number of steps. Moreover, since each intermediate global state contains at least one valid enabled transition, at the end of the algorithm, the final global state must be $S_{H}$. So pex thus constructed is a parallel execution sequence for $S_{H}$. (Please refer to the full paper [SL97] for details.) Hence we have $\mathbf{S N R} \mathbf{R}_{H} \subseteq \mathbf{P N R}_{H}$, and thus $\mathbf{P N R}_{H}=\mathbf{S N R} \mathbf{N R}_{H}$. Since $\mathbf{S} \mathbf{N R}_{H}$ is exactly the set of non-progress states in $R$, we have the following result on fault coverage of $\mathbf{P R}_{H}$.

Theorem 3 Given $S_{R} \in \mathrm{R}_{R}, S_{R}$ is non-progress global state in $R$ iff $S_{H}=\alpha\left(S_{R}\right)$ is a parallel non-progress global state in $H$.

\subsection{Discussion}

The parallel step technique described in this section was adapted from the simultaneous reachability analysis method in [OU94] to fit the context of protocol composition. Similar to [SU96], we can use the "sleep-set" concept [GW93, GW94] to further eliminate redundant transitions in computing the set of parallel progress vectors. We can also correlate transitions from different processes, as was done in fair reachability analysis [RW82, GH85, LM96a, LM96b]. Doing so might result in fewer global states, but the computation in each global state becomes more elaborate.

In this paper, we assume that the component protocols have the required progress property. If the composite protocol has non-progress global states, then it is most likely that the composition constraints are not consistent with each other. Hence in analyzing error scenarios, we should focus on the set of constraints involved. Note that not all the non-progress global states are semantically incorrect. For example, if one action inhibits the other and that action corresponds to an exception in the protocol, the protocol may halt in response to that exception. So it is up to the designer to decide whether a non-progress global state is acceptable or not. However, the imposed constraints are not the only cause for non-progress in the composite protocol. This point is more subtle. Recall that in our model, a send statement is blocked if the destination channel is full. In the composite protocol, the bound on a channel might be enlarged. So it is possible that a send action that is not enabled in the original component protocol becomes enabled in the composite protocol. So a process may exhibit new behaviors after the composition. These new behaviors, together with their interactions may also cause non-progress in the composite protocol.

Last but not least, even though we presented our technique in the context of two component protocols $P$ and $Q$, it can be easily extended to handle cases with more than two component protocols. Furthermore, our technique does not require that all component protocols have the same number of processes, nor does it require that the composition of processes be fixed w.r.t the indices of the processes in each component protocol. All is required is that at most one process from each component protocol can participate in each site in the composite protocol. However, not every component protocol is required to participate in the composition for a site. What we need is a composition schema that describes which process from which component protocol is needed to participate at each site. Once the schema is given, we can define composition constraints for each site with more than one process, and the rest of the work can proceed as described above. In the next section, we will give an example in this general setting. 


\section{EXAMPLE}

Consider a network of four sites shown in Figure 1(a). We want to design a data transfer protocol in which site 1 first establishes connection with sites 2, 3 and 4 and then transfers a sequence of data items to them. Site 1 send the items directly to 2 and 4 and site 2 forwards the data items to 3. We want a stop\&wait protocol in which 1 sends the next data item only after all sites have received the previous data item. Finally, site 1 may send a disconnect message at any time after the connection establishment to break the connection.

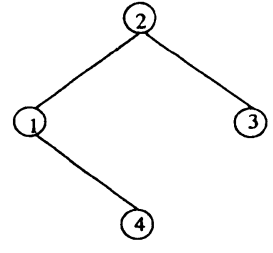

(a)

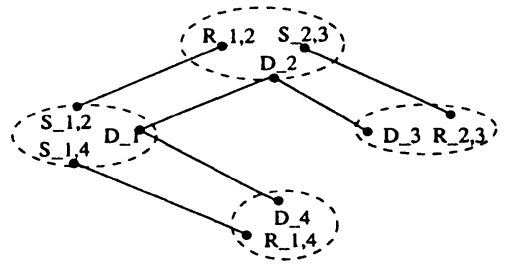

(b)

Figure 1 Topology for the data transfer protocol.
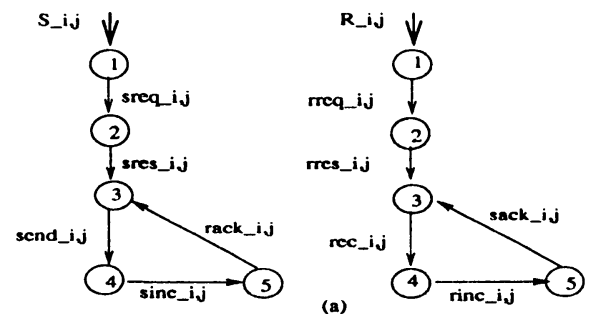

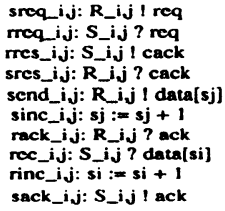

(a)
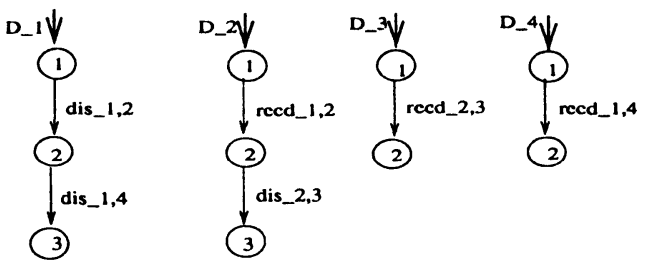

dis_1,2: D_2 ! dis

dis_1,4: D_4 I dis

recd_1,2: D_1 ? dis

rocd_1,4: D_1? dis

dis_2,3: D 3 ! dis

rocd_2,3: D_2? dis

(b)

Figure 2 Data transfer component protocols.

Figure 2(a) gives a stop\&wait protocol $\left(S_{i, j}, R_{i, j}\right)$ with $S_{i, j}$ at site $i$ as the sender and $R_{i, j}$ at site $j$ as the receiver. Figure 2(b) gives a disconnect protocol in which 1 simply sends a disconnect message to 2 and 4 , and 2 forwards it to 3 . We will design protocols using four component protocols: $\left(S_{1,2}, R_{1,2}\right),\left(S_{1,4}, R_{1,4}\right),\left(S_{2,3}, R_{2,3}\right)$ and $\left(D_{1}, D_{2}, D_{3}, D_{4}\right)$ (see Figure $\left.1(\mathrm{~b})\right)$.

As a simpler case, we first compose $\left(S_{1,2}, R_{1,2}\right)$ and $\left(S_{1,4}, R_{1,4}\right)$ with a synchronization constraint $\left(\operatorname{send}_{1,2}, \operatorname{send}_{1,4}\right)$ at site 1 to ensure that the first data item is sent after connection with 
both 2 and 4 has been established, and subsequent data items are sent only after acknowledgements from both 2 and 4 are received for the previous data item. For the composite protocol built by the algorithm in section 3, the standard reachability analysis explores 126 states, the partial order method in [HGW92] finds 81 states, whereas our method has only 9 reachable states (here we view inc $i, j$ as an internal action; otherwise, the number of states are unbounded). In fact, our method explores 9 states irrespective of the number of receivers.

The next protocol with all four sites is obtained by combining all four protocols with seven more constraints: (1) An ordering constraint $\left(\operatorname{rec}_{1,2}, \operatorname{send}_{2,3}\right)$ on 2 to ensure that a data item is forwarded to 3 only after it has been received from 1 ; (2) An order constraint $\left(\right.$ rack $_{2,3}$, sack $_{1,2}$ ) on 2 to ensure the stop\&wait discipline w.r.t 1 and $3 ;(3)$ Two ordering constraints $\left(\right.$ sres $_{1,2}$, dis $\left._{1,2}\right)$ and $\left(\right.$ sres $_{1,4}$, dis $\left._{1,4}\right)$ on site 1 to ensure 1 can send a disconnect message only after connection setup; (4) Three inhibition constraints $\left(\right.$ dis $\left._{1,2}, \operatorname{send}_{1,2}\right),\left(\operatorname{dis}_{1,4}, \operatorname{send}_{1,4}\right)$ and $\left(\right.$ dis $_{2,3}$, send $\left._{2,3}\right)$ to ensure no more data items are to be sent after the disconnect message is sent. These constraints allow the messages that have already been sent to be received and acknowledged. Although the final composite protocol is a complex one, our method explores only 47 reachable states.

\section{CONCLUSION AND FUTURE WORK}

In this paper, we studied the problem of validating protocol composition for progress based on the set of component protocols and a set of composition constraints. By encoding the constraints into the processes of component protocols and the analysis algorithm, we are able to perform parallel step state exploration for the composite protocol without constructing it explicitly. As a result, we are able to perform validation for the composite protocol in a significantly reduced global state space. As far as we know, this is the first attempt to adapt existing state reduction techniques to protocol composition.

However, we have just scratched the surface in this direction. First, the composite protocol construction algorithmgiven in Section 3 may not be the most efficient one, and the $R_{i}$ constructed may not be the minimum state machine for the composite process. How to build a minimum state composite process is an interesting problem that requires further study. Second, It would be interesting to investigate other encoding schemes to fit the partial order techniques so that more general properties can be validated. We also want to include more constraint types to allow more flexible compositions. Finally, we plan to implement the parallel step method and experiment it with complex examples.

\section{Acknowledgement}

The authors would like to thank Raymond E. Miller and Jun-Cheol Park for their constructive comments on the earlier drafts of this paper.

\section{REFERENCES}

[CR93] L. Cacciari and O. Rafiq, "On Improving Reduced Reachability Analysis," Proc. FORTE'92, Perros-Guirec, France, October 13-16, 1992, pp. 137-152. 
[CGL85] C.H. Chow, M.G. Gouda and S.S. Lam, "A Discipline for Constructing Multi-Phase Communicating Protocols," ACM Trans. Comput. Syst., 3(4), 1985, pp. 315-343.

[CM86] T.Y. Choi and R.E. Miller, "Protocol Analysis and Synthesis by Structured Partitions", Computer Networks and ISDN Systems, 11, 1986, pp. 367-381.

[GH85] M. Gouda and J.Y. Han, "Protocol Validation by Fair Progress State Exploration," Computer Networks and ISDN Systems, 9, 1985, pp. 353-361.

[GW93] P. Godefroid and P. Wolper, "Using Partial Orders for the Efficient Verification of Deadlock Freedom and Safety Properties, ${ }^{n}$ Formal Methods in System Design, 2(2), 1993.

[GW94] P. Godefroid and P. Wolper, "A Partial Approach to Model Checking," Information and Computation, 110(2), 1994, pp. 305-326.

[HGW92] G. Holzmann, P. Godefroid and P. Wolper, "Coverage Preserving Reduction Strategies for Reachability Analysis," PSTV'92.

[II83] M. Itoh and H. Ichikawa, "Protocol Verification Algorithm Using Reduced Reachability Analysis, ${ }^{n}$ Trans. IECE of Japan, E66(2), 1983, pp. 88-93.

[Lin88] H.A. Lin, "A Methodology for Constructing Communication Protocols with Multiple Concurrent Functions," Distributed Computing, 3(1), 1988, pp. 23-40.

[Lin91] H.A. Lin, "Constructing Protocols with Alternative Functions," IEEE Transactions on Computers, 40(4), 1991, pp. 376-386.

[LT93] H.A. Lin and C.L. Tarng, "An Improved Method for Constructing Multiphase Communications Protocols, ${ }^{n}$ IEEE Transactions on Computers, 42(1), 1993, pp. 15-26.

[LM96a] H. Liu and R. Miller, "Generalized Fair Reachability for Cyclic Protocols, ${ }^{n}$ IEEE/ACM Transactions on Networking, 4(2), April 1996, pp. 192-204.

[LM96b] H. Liu and R.E. Miller, "An Approach to Cyclic Protocol Validation," Computer Communications, 19(14), 1996, pp. 1175-1187.

[LM96c] H. Liu and R.E. Miller, "Partial-Order Validation for Multi-Process Protocols Modeled as Communicating Finite State Machines," Proc. ICNP'96, Oct. 29 - Nov. 1, 1996, pp. 76-83.

[OU94] K. Özdemir and H. Ural, "Deadlock Detection in CFSM Models via Simultaneously Executable Sets," ICCI'94, Peterborough, Ontario, Canada, May 1994, pp. 673-688.

[P93] D. Peled, "All from One, One for All: On Model Checking Using Representatives," CAV'93.

[P94] D. Peled, "Combining Partial Order Reduction with On-the-fly Model-Checking," CAV'94.

[RW82] J. Rubin and C.H. West, "An Improved Protocol Validation Technique," Computer Networks, 6, 1982, pp. 65-73.

[S93] G. Singh, "A Compositional Approach for Designing Protocols," Proc. ICNP'93, San Francisco, CA, October 19-22, 1993, pp. 98-105.

[S94a] G. Singh and M. Sammeta, "On the Construction of Multiphase Protocols," Proc. ICNP'94, Boston, MA, October 25-28, 1994, pp. 151-158.

[S94b] G. Singh, "A Methodology for Constructing Communication Protocols," Proc. ACM SIGCOMM'94, August 31 - September 2, 1994, London, U.K., pp. 245-255.

[SL97] G. Singh and H. Liu, "Validating Protocol Composition for Progress by Parallel Step Reachability Analysis," in preparation.

[SU96] H.v.d. Schoot and H. Ural, "Protocol Verification by Leaping Reachability Analysis," Proc. IC3N'96, Rockville, MD, USA, October 16-19, 1996, pp. 334-339.

[V90] A. Valmari, "A Stubborn Attack on State Explosion," Proc. CAV'90.

[YG82] Y.T. Yu and M.G. Gouda, "Deadlock Detection for a Class of Communicating Finite State Machines, ${ }^{n}$ IEEE Transactions on Communications, 30(12), 1982.

[ZB86] J.R. Zhao and G.v. Bochmann, "Reduced Reachability Analysis of Communication Protocols: a New Approach," Proc. PSTV'86, pp. 243-254. 